\title{
Indoor Taxi-Cab: Real-Time Indoor Positioning and Location-Based Services with Ekahau and Android OS
}

\author{
Caner Kilinc, Seraj Al Mahmud Mostafa, Raihan U1-Islam, Kashif Shahzad, and Karl Andersson \\ Pervasive and Mobile Computing Laboratory \\ Luleå University of Technology \\ SE-931 87 Skellefteå, Sweden \\ \{kilcan-0@student., sermos-9@student., raiuli-0@student., kassha-0@student., karl.andersson@\}ltu.se
}

\begin{abstract}
Positioning and routing in outdoors environment is still challenging especially in complex buildings, where a number of buildings are combined with tunnels and bridges, and the GPS signal is unreachable. Looking for a particular room in an unfamiliar huge indoor environment or a product in an enormous store is time wasting and a real life problem that everybody faces on a daily basis. This paper presents a solution using the Ekahau positioning system and Android OS with an intermediary server, which acts between these two systems to provide actual room level positioning by a mathematical modeling technique. The system also provides the shortest path to a target destination by using Dijkstra's search algorithm. The optimal distance between the locations is modeled based on the Taxi-cab geometry distance definition for the mobile clients. Additionally, the users can also display the shortest path for items in the vicinity such as coffee machines, etc. The proposed solution has been evaluated in a real life setting at basement floor level.
\end{abstract}

Keywords: Indoor Positioning; Indoor Location-based Services; Ekahau; Mathematical Modelling; Dijkstra's Search Algorithim; Taxi-Cab geometry; Android OS

\section{INTRODUCTION}

In 2013, the number of connected mobile devices reached 10 billion [1] and the amount of smart phones usage reached to 1.4 billion: 798 million of them are Android based devices and 294 million are iPhone [2]. Nowadays, Billions of users are using Global Navigation Satellite System (GNSS) such as Global Positioning System (GPS) [3], which is the most well-known. GPS technology relies on signals from multiple satellites and employs a triangulation process to determine physical locations with an accuracy of about 33 feet (10 meters).

Many business and private zones are already covered by wireless networks for connecting laptops, PDAs and mobile phones. These devices could be tracked simply by adding enabling software. However, GPS technology cannot be used effectively in dense urban areas, due to blind spots, weak radio signals, and other signal interference factors. The lack of GPS-based location services in huge buildings reinforce to fuse smartphones and Wi-Fi signal strength to enable positioning using Euclidean geometry distance calculation. This paper presents Taxi geometry for the distance computation, which has so far, to best of our knowledge, not been used for this purpose. The reason is that Taxi geometry comes by and takes advantage of lower code complexity such as decreased memory consumption and execution time of the computation.

Indoor positioning systems (IPSs) with accurate, reliable and real-time location updates has always been significantly challenging, where there are a lot of physical barriers depending on radio signal absorption or obstruction in a large indoor space with lots of corridors, floors, rooms and objects. For IPSs there are several systems that have been provided including RFID, WLAN, UWB, ZigBee, infrared and ultra sounds, etc. Lots of studies have been done and remarks that specific calibration techniques are needed to be able to obtain accurate positioning and mapping results related with site characteristics. Previous work provide an overview of indoor positioning techniques [4][5][6].

The following techniques are available:

- Radio Map (RM): RM definition provides the variations of the RSS values from the AP's. It also gives detailed information on cell size and the distances of APs. However, using the RM definition database helps in accuracy to track devices. The RSS values are immediately compared with the recent signal strength while a moving device receives any signal from an AP [7][8]. RM definition table does not store any new data. It only compares the new received values from the device with the RSS locations. So, it becomes quite easy to detect user accurate location immediately.

- Motion Estimation: In the literature, a number of object motion estimation methods are described and used [4] e.g. the radio-frequency (RF) based RADAR system: Radar is a sensor, which can be placed on walls in order to gather Wi-Fi signal strength information at multiple locations to triangulate the object's coordinates [9][10].

These both given techniques are used together by Crowdsourcing Localization [11][12][13]. Last but not least, Simultaneous Location and Mapping (SLAM) can be given as an additional example, which is an active research project for more than 20 years [14][15]. 
A number of IPSs have been developed based on these fundamental techniques. Each system takes advantage of a particular positioning technology or combining some of these technologies and [5] introduces and evaluates different commercially available and research-oriented IPSs.

As a solution to the addressed problems and challenges, this paper presents Indoor Taxi-Cab for real time indoor positioning and services. Indoor Taxi-Cab uses Ekahau Positioning Engine (EPE) as a tool to obtain users' $(x, y)$ coordinates by using the signal strength mapping and the motion estimation techniques in a composition. The determined coordinates are handled at an intermediate server where the users' room level location is determined and based on the demand a shortest path is provided, with Dijkstra's algorithm based on Taxi-Cab geometry distance definition, for a certain destination or a closest item e.g. Xerox machine or Coffee machine.

EPE offers a practical 802.11 b-based Wireless LAN indoor positioning system that requires no proprietary hardware additions to the network and obtains clients' location from Ekahau tags which is an RFID (Radio Frequency Identifier) [16] that transmits coordinates of the client to the EPE over Wi-Fi. EPE is designed to be used as a WLAN positioning server, or integrated with vertical software applications or hardware. It is a powerful Java ${ }^{\mathrm{TM}}$ software component that provides user and asset coordinates ( $\mathrm{x}, \mathrm{y}$, floor) and tracking features to client applications [17].

EPE services have not yet been provided for Microsoft's and Apple's mobile operating systems. Therefore, an additional Java based intermediary server allows us to provide the positioning and location based services to any kind of mobile users. In the proposed system, an Android application is implemented and evaluated as a mobile client.

Furthermore, the intermediate server determines users' exact room level location based on the $(x, y)$ coordinates with a mathematical modeling technique which increases Ekahau's capability while decreasing the error rate by room level location detection. Based on the Dijkstra's Search Algorithm the shortest path is computed to the requested target destination [18][19] where each room or each nearest item is defined as a node. Dijkstra's algorithm provides a set of nodes/room names as an output. Those rooms need to be visited to reach the destination and it is eventually the shortest path. The intermediate server transmits to the Android Client and updates in real time whenever users' coordinates are changed.

Furthermore, the Indoor Taxi-Cab solution provides an optimal route to a desired destination. At this phase, TaxiCab geometry is exploited which is unique. Taxicab geometry was defined by E. F. Krause [20] and is very similar with the Euclidean coordinate geometry. Definition of points and lines are the same as well as the angles are measured in the same way [21]. The difference is that computation of distances which is not calculated as the crow flies, but as a taxi would drive. The number of blocks horizontally and vertically considered in this computation to get from a certain point $A$ to point $B$. The same destination computation technique is used while determining the distance across the rooms and corridors. Also it is used while drawing an optimal route for the users due to the user cannot pass through the wall. Therefore, they have to follow the corridors like a taxi follows the streets to reach a targeted destination. Additionally, the user also can request a shortest path for the nearest item via the Android application, which will also be handled in the intermediary server.

The remainder of the paper is structured in the following way. Section 2 indicates related work, while Section 3 describes our system architecture. Section 4 concludes, provides evaluation and discusses the results.

\section{RELATED WORK}

Recently, there is striking development on the real time indoor positioning. Feldman et al. [23] proposed a model for location estimation using a triangular method. They used RSSI (radio signal strength indicator) and the distance between sender and the receiver. Gu et al. [5] and Lui et al. [6] provided survey studies of indoor positioning systems for wireless personal networks. Shin et al. [7] introduced an indoor user tracking system. The system constructs a topological map with Wi-Fi signal calibrations, assigns semantically meaningful labels into the map, and estimates the semantic location of the user based on the current Wi-Fi signal strength observation. Eventually, the proposed system was the first Wi-Fi fingerprinting-based SLAM implementation. Lau et al. [7] presented the Density-based Cluster Combined Localisation Algorithm that uses the unsupervised technique to recognise meaningful locations. The results prove that the DCCLA is able to detect fingerprints of $\mathrm{Wi}-\mathrm{Fi}$ signal strengths and does not require users' input. Godana et al. [9] developed Radar technology in order to monitor and operate remotely human activity in the form of a set of Doppler frequency. Regarding the results in the study, the system is able to estimate the velocity profile of the person's translational movement with less than $10 \%$ error. Bahl et al. [10] performed a similar study and presented results. Rai et al. [11] proposed the Zee system, which makes the calibration zero-effort, by enabling training data to be crowd sourced without any additional location input from users. Rai's experimental results highlight that WiFi-based positioning techniques, both fingerprinting-based and modeling-based, are able to perform accurate localization, when crowd sourced data is using Zee. Ali et al. [13] implemented algorithms such as the Super Resolution Matrix Pencil (MP) algorithm, which gives a better estimation for TOA (time of arrival), as the TOA has superior performance over the Fourier transform technique, so it is important to estimate the TOA accurately to identify any object. Johnson et al. [18] examine the Dijkstra's shortest path algorithm and evaluated at [19] whether it is suitable for iterative routing for the operation of culler multi-hop networks based on various scenarios. 
The study shows that better delay and cost performance can be achieved by the iterative algorithm.

Finally, Li et al. [20] studied privacy-preserving location sharing in mobile online social networks. They observed that users' real fake identities could potentially be leaked to location service providers. Li et al. [20] therefore proposed an improved mechanism that employs dummy queries and a private set intersection protocol to prevent the online service network service and location service providers from learning individual information from each other.

\section{SySTEM ARCHITECTURE}

The implementations and experiments are carried out at the university campus, in a basement corridor at a building. Our Indoor Taxi-Cab system architecture consists of three different components:

1. Ekahau Server (Ekahau Positioning Engine)

2. An Intermediate Server

3. An Android application and Ekahau Tag

as shown in Figure 1.

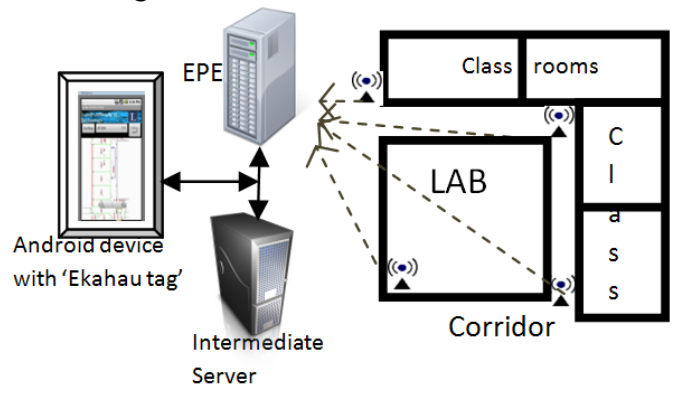

Figure 1: Indoor Taxi-Cab Overall System Architecture.

Ekahau tag is carried around by the Android Clients. The tag measures the Wi-Fi signal strengths from a number of different Wireless Access Points and sends the position of the strongest signal to the Ekahau server to determine accurate $(x, y)$ position of the users. The Ekahau server continuously obtains the user's position from the Ekahau tag and sends it to the Intermediate server in real time.

The Intermediate server has been developed by us in order to determine the user's room level position on a relevant indoor map. It also provides an optimal route to a certain target destination which can be a room or an item, e.g. nearest a copy or coffee machine. The system works as the following.

First, all rooms and corridors are defined by an individual set of unique $x$ and $y$ coordinates, e.g. room $\mathrm{A}$ is modeled as $\left(x_{a}, y_{a}\right)$.

As shown in Figure 2 any room can be identified by a set of unique $x$ and $y$ coordinates for instance: a rectangle room $\mathrm{A}$ is defined as follows:

$$
A=\left\{\left(x_{a}, y_{a}\right) \mid x_{1} \leq x_{a} \leq x_{2}, y_{1} \leq y_{a} \leq y_{2}, a \in \mathbb{N} \text { and } x, y \in \mathbb{R}\right\}
$$

Note in the figure that the distances between any of the rooms and corridors are abstract to provide a crystal clear picture.

Whenever measured a pair of axes e.g. $\left(x_{u}, y_{u}\right)$ are obtained for a user from the Ekahau server, function $f$ identifies the exact room number where the Android client is located and that is defined as below for the rectangular rooms;

$f\left(x_{a}, y_{a}\right)=\left\{\begin{array}{l}A, x_{1}<x_{a}<x_{2}, y_{1}<y_{a}<y_{2} \\ B, \quad x_{1}<x_{b}<x_{2}, y_{1}<y_{b}<y_{3}\end{array}\right.$

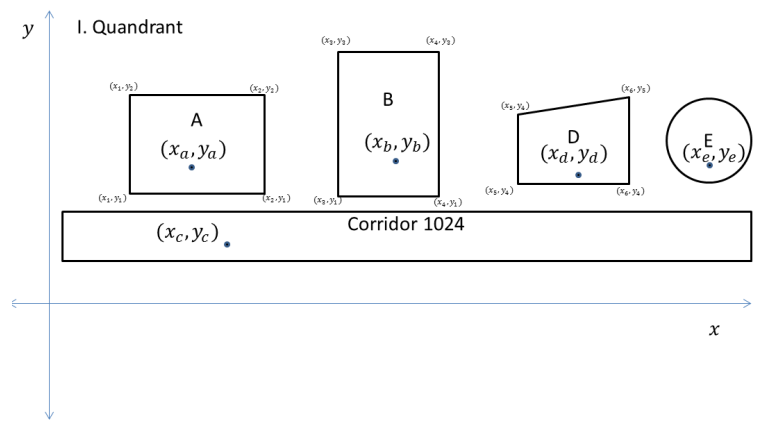

Figure 2: Mathematical Modeling for Room Level Location Determination

Furthermore, a circular room, with center $C=(h . k)$ and radius $r$ is modeled as follow;

$$
(x-h)^{2}+(y-k)^{2}=(r)^{2}
$$

This modeling is done and it can be done for any kind of room regardless its shape, due to that there are lots of wellknown linear calculation techniques and formulas in analytic geometry to determine whether a certain per of axes is in a geometric shape or not.

Even if the $(x, y)$ coordinates of the user have been erroneously detected, e.g. by one meter, there is a higher possibility that the user is still in the same room or corridor. Therefore, the mathematical model used increases Ekahau's capability while decreasing the error rate by room level location detection. Additionally, one or a number of key points are identified in each room and corridor in order to be used at the next step.

Once the user's current located room is determined then a small circle is sketched, on the relevant area of the map. Then an optimal walking route, from the current location to the targeted destination, is computed.

Dijkstra's algorithm is used in order to find the shortest walking path and the algorithm works according to the following. First, the current location is set as the starting point/node and set to zero as the value (starting point is zero) of the current node, which is shown in Figure 3. 
Second, the current node will be the closest unvisited neighbouring node of the current node. It is worthwhile to keep in mind that the distance between the nodes is calculated based on Taxi-Cab geometry destination definition, which is explained later on in this section.

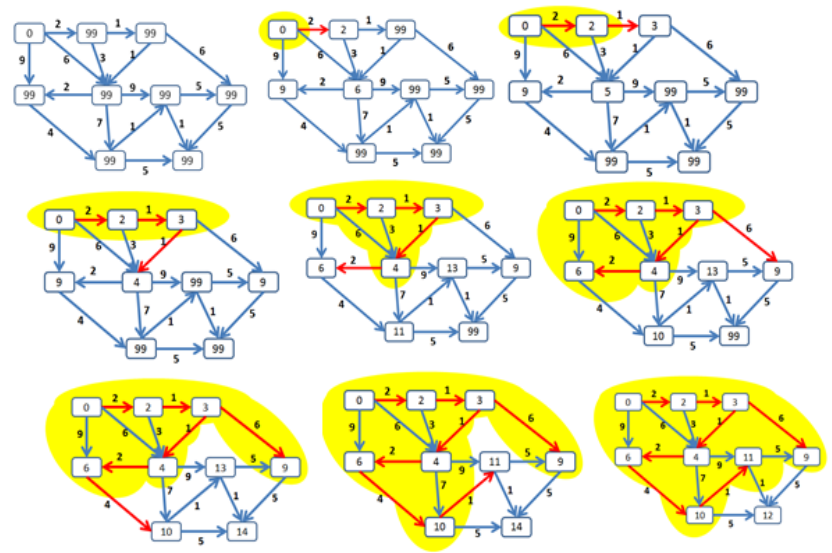

Figure 3: Dijkstra's Algorithm

On the current node, the distance to every unvisited node, which is directly connected to the current node, is considered by calculating the total distance between an unvisited node and the value of the current node. Afterwards, the unvisited node is marked with this calculated value, if it is less than its current value. Note that, if the path to it through the current node is shorter than the previously known paths, the node is relabelled. After the distances are determined to each neighbouring nodes, the current node is coloured as visited and select the closest unvisited node as the current node.

Dijkstra's algorithm also defines two sets of nodes: OPEN and CLOSED. An OPEN node means that the set of vertices to process while a CLOSED node includes vertices already processed. Therefore, the coloured nodes will not be revisited or returned to.

The searching process for selecting the neighbouring nodes with the shortest distances continues colouring the current node as visited and moving onto the closest unvisited neighbour node until the target destination node is coloured as visited. Once the target destination node is coloured as visited, that means the shortest path is determined and as output a list of nodes in a visiting order is obtained, which needs to be visited one by one to reach to the target destination in the given order.

In order to draw a route to the desired destination, Taxicab geometry is exploited as follows; Figure 4 gives an example where a user is standing at a point $K\left(x_{1}, y_{1}\right)$ and wants to go to point $D\left(x_{3}, y_{2}\right)$, based on this demand as explained above, first the shortest path is determined with Dijkstra's algorithm. In this case the output of the Dijkstra's algorithm is

$\phi=\{$ Red Corridor, Grey Corridor, Green Corridor $\}$ where $\phi$ is a set of rooms and corridors (represented as nodes in the algorithm).

The identified key points for the corridors are as following; $K\left(x_{1}, y_{1}\right)$ is in blue horizontal corridor, $P_{1}\left(x_{2}, y_{1}\right)$ and $P_{2}\left(x_{2}, y_{2}\right)$ in grey vertical corridor, and $D\left(x_{3}, y_{2}\right)$ is in green horizontal corridor

where $\left|K P_{1}\right| \perp\left|P_{1} P_{2}\right| \perp\left|P_{2} D\right|$ and $\left|K P_{1}\right| \|\left|P_{2} D\right|$

hence $L\left(x_{3}, y_{1}\right)$ can be found where $|K L| \perp|D L|$.

According to Pythagorean Theorem in Euclidean geometry, the shortest path between $K\left(x_{1}, y_{1}\right)$ and $D\left(x_{3}, y_{2}\right)$ has been calculated by

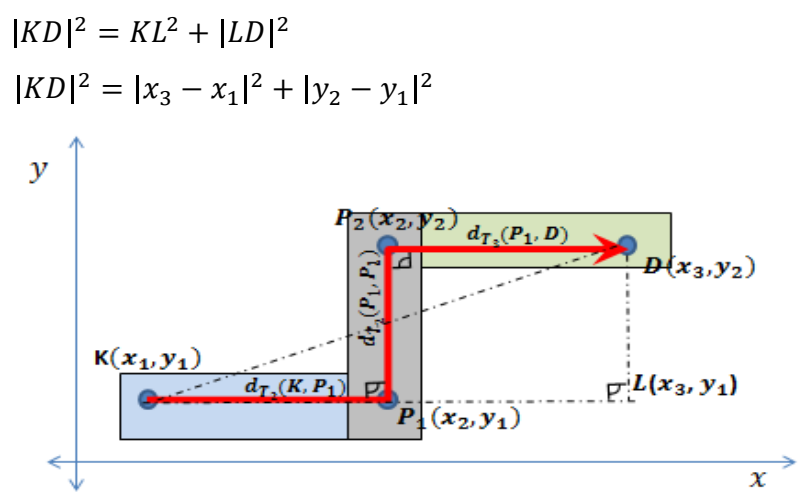

Figure 4: Taxi-Cab Geometry Distance Definition

The significant difference in Taxi-Cab geometry that the shortest path between $K\left(x_{1}, y_{1}\right)$ and $D\left(x_{3}, y_{2}\right)$, is shown with a red route on the figure above, has been defined by

$$
\begin{aligned}
& |K D|=\left|K P_{1}\right|+\left|P_{1} P_{2}\right|+\left|P_{2} D\right| \\
& |K D|=d_{T_{1}}\left(K, P_{1}\right)+d_{T_{2}}\left(P_{1}, P_{2}\right)+d_{T_{3}}\left(P_{2}, D\right) \\
& |K D|=\left|x_{2}-x_{1}\right|+\left|y_{2}-y_{1}\right|+\left|x_{3}-x_{2}\right|
\end{aligned}
$$

Due to the given definition Taxi-Cab distances between the rooms and corridors are computed and stored in the Intermediate server. The key points are linked as a walking path based on the request.

Finally, the optimal route is determined and plotted on the map and it is transmitted to the Android client. Figure 5 shows an additional feature that a user can request for a nearby item, while Figure 6 shows the user having selected a particular room name to get the optimal route. Finally, Figure 7 shows a typical response. 


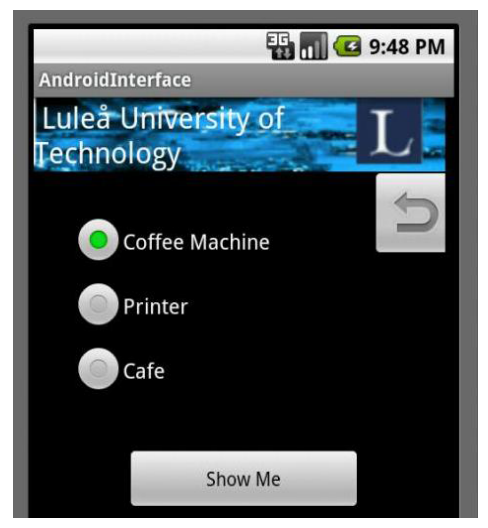

Figure 5: User can request for a nearby item

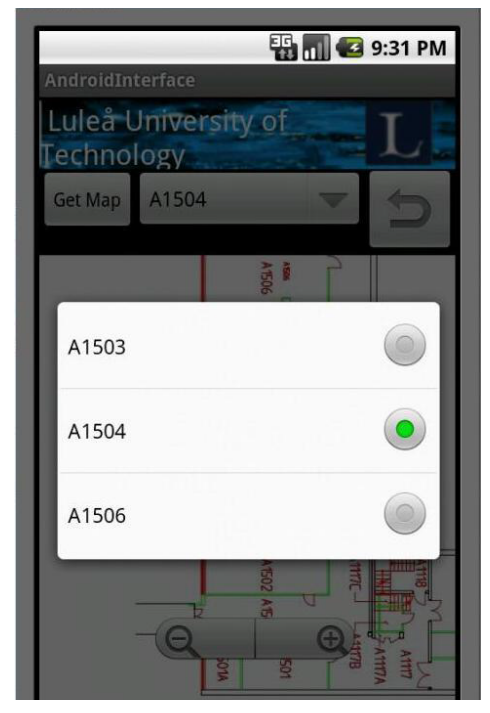

Figure 6: User requests for a destination

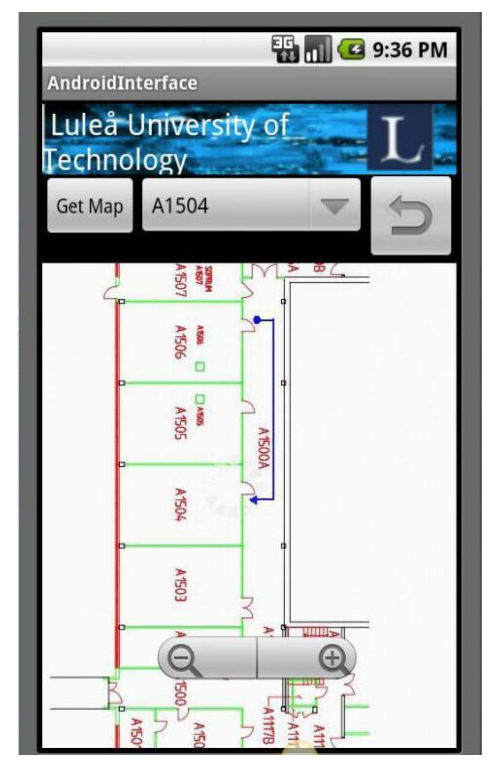

Figure 7: The shortest path for a certain destination

\section{CONCLUSION AND FUTURE WORK}

We provided an efficient system for indoor positioning. Users of the system were pleased and excited by having such an application where they could be helped by our Indoor Taxi-Cab system to know the exact direction and location in a complicated indoor environment where a number of buildings are linked via tunnels and bridges.

The proposed system relies on a commercially launched Ekahau indoor positioning product while obtaining the users' coordinates based on Wi-Fi radio signal strength measurements. Unfortunately, the system inherits the Ekahau's limitations related to performance and pricing. Furthermore, the cost of mapping the campus along with licenses can be quite high.

Our future work includes testing alternative open source systems to obtain the users' coordinates. Also, we intend to extend the system and to develop our own Wi-Fi positioning engine. Finally, we intend to port our system to iOS and Windows 8

In conclusion, the main problem is to balance the performance load, how to distribute processing power between the clients and server, and how to utilize limited bandwidth.

\section{ACKNOWLEDGMENT}

Part of this work is a result of the NIMO (Nordic Interaction and Mobility Research Platform) project [24] supported by the EU Interreg IVA North program.

\section{REFERENCES}

[1] http://techcrunch.com/2012/02/14/the-number-ofmobile-devices-will-exceed-worlds-population-by2012-other-shocking-figures/

[2] http://www.businessinsider.com/15-billionsmartphones-in-the-world-22013-2

[3] http://en.wikipedia.org/wiki/Global_Positioning_Syste $\mathrm{m}$

[4] P. Bissig, R. Wattenhofer, and S. Welten. (2013). A Pocket Guide to Indoor Mapping. Proc. WPNC

[5] Y. Gu, A. Lo, and I. Niemegeers. (2009). A Survey of Indoor Positioning Systems for Wireless Personal Networks. IEEE Communications Surveys \& Tutorials, IEEE 11(1):13-32

[6] H. Liu, H. Darabi, P. Banerjee, and J. Liu. (2007). Survey of Wireless Indoor Positioning Techniques and Systems. IEEE Transactions on Systems, Man, and Cybernetics, Part C: Applications and Reviews 37(6):1067-1080

[7] M. Ficco, C. Esposito, and A. Napolitano. (2014). Calibrating Indoor Positioning Systems with Low Efforts. IEEE Transactions on Mobile Computing 13(4):737-751

[8] S. L. Lau, Y. Xu, and K. David. (2011). Novel Indoor Localisation using an Unsupervised Wi-Fi Signal Clustering Method. Proc. FutureNetw 
[9] B. Godana, G. Leus, and A. Barroso. (2010). Estimating Indoor Walking Velocity Profile using a Software Radio-based Radar. Proc. SENSORDEVICES

[10] P. Bahl and V. Padmanabhan. (2000). RADAR: An inbuilding RF-based User Location and Tracking System. Proc. IEEE INFOCOM

[11] A. Rai, K. Chintalapudi, V. Padmanabhan, and R. Sen. (2012). Zee: Zero-effort Crowdsourcing for Indoor Localization. Proc. Mobicom

[12] M. Lee, H. Yang, D. Han, and C. Yu. (2010). Crowdsourced Radiomap for Room-level Place Recognition in Urban Environment. Proc. IEEE PERCOM Workshops

[13] A. Ali and A. Omar. (2005). Time of Arrival Estimation for WLAN Indoor Positioning Systems using Matrix Pencil Super Resolution Algorithm. Proc. WPNC

[14] H. Durrant-Whyte and T. Bailey. (2006). Simultaneous Localization and Mapping: Part I. IEEE Robotics \& Automation Magazine 13(2):99-110

[15] T. Bailey and H. Durrant-Whyte. (2006). Simultaneous localization and mapping (SLAM): Part II. IEEE Robotics \& Automation Magazine 13(3):108-117

[16] http://en.wikipedia.org/wiki/Radiofrequency_identification

[17] http://www.ekahau.com/

[18] D. Johnson. (1973). A Note on Dijkstra's Shortest Path Algorithm. Journal of the ACM 20(3):385-388

[19] D. Johnson, D. Maltz, and J. Broch. (2001). DSR: The Dynamic Source Routing Protocol for Multi-hop Wireless Ad Hoc Networks. Ad hoc networking, Addison-Wesley Longman Publishing, pp. 139-172

[20] J. Li, J. Li, X. Chen, Z. Liu, and C. Jia. (2014). MobiShare+: Security Improved System for Location Sharing in Mobile Online Social Networks. Journal of Internet Services and Information Security 4(1):25-36

[21] E. Krause. (1973). Taxicab geometry. The Mathematics Teacher 66(8):695-706

[22] E. Krause. (1987). Taxicab geometry: An adventure in non-Euclidean geometry, DoverPublications.com

[23] S. Feldmann, K. Kyamakya, A. Zapater, and Z. Lue. (2003). An Indoor Bluetooth-based Positioning System: Concept, Implementation and Experimental Evaluation. Proc. International Conference on Wireless Networks

[24] NIMO Project (2014). Available at http://www.nimoproject.org 